\title{
Further Development of Velocity-based Airborne TPA: Scan \& Paint TPA as a Fast Tool for Sound Source Ranking
}

\author{
Daniel Fernandez Comesana, Andrea Grosso, Hans-Elias De Bree and Jelmer Wind \\ Microflown Technologies BV
}

Keith Holland

ISVR

\begin{abstract}
The interior noise of a car is a general quality index for many OEM manufacturers. A reliable method for sound source ranking is often required in order to improve the acoustic performance. The final goal is to reduce the noise at some positions inside the car with the minimum impact on costs and weight. Although different methodologies for sound source localization (like beamforming or p-p sound intensity) are available on the market, those pressure-based measurement methods are not very suitable for such a complex environment.
\end{abstract}

Apart from scientific considerations any methodology should be also "friendly" in term of cost, time and background knowledge required for post-processing.

In this paper a novel approach for sound source localization is studied based on the direct measurement of the acoustic particle velocity distribution close to the surface. An airborne transfer path analysis is then performed to rank the sound pressure contribution from each sound source.

The method called "Scan \& Paint TPA" makes use of only one probe that is swept along the surface. The reciprocal transfer functions are measured by a second sweep with the same probe and a monopole sound source in the driving position. A new methodology for applying "Scan \& Paint TPA" in a complex acoustic environment is given along with an experimental validation in a car interior.

\section{INTRODUCTION}

One common problem in vehicle interior noise is reducing the sound pressure level at certain positions. Following the common ground between most widespread techniques, the car interior can be discretized into several 'panels'. Then, their degree of 'contribution' should be defined in order to rank which panels has a stronger influence on causing the sound pressure at the evaluated position. This problem is normally referred to as "Panel Contribution Analysis". In the technical literature, several experimental techniques can be found that address this problem. Most commonly used methods are window-based techniques $[\underline{1}, \underline{2}]$, intensity measurements [3] , laser scanning vibrometry measurements [4], beam forming [] $]$ and holographic technologies [ $[6,7]$ using sensor arrays.

Other works on airborne transfer path analysis for velocity based methods showed the potential of combining multichannel volume velocity measurements with acoustic transfer paths for predicting sound pressure at a certain reference position $[\underline{8}, \underline{9}, \underline{10}]$. Nonetheless, there is a lack of evidence if velocity-based scanning measurement techniques could be also applied to complex industrial applications.

Already from the late 1980 's scanning methods have been introduced for mapping stationary sound fields [11]. Recent works have introduced a novel scanning method called "Scan $\&$ Paint" $[12,13,14]$ for measuring sound pressure, particle velocity, intensity, sound absorption and acoustic impedance in an efficient way. The properties of the sound field are determined and visualized via the following routine: while 
the probe is moved slowly over the surface, pressure and velocity are recorded and, at the same time, a video image is captured. Next, all data is processed. At each time interval, the video image is used to determine the location of the sensor. The absolute position of the probe is unknown, only the $2 \mathrm{D}$ coordinates relative to the background image are computed. Then, an acoustic color plot is generated.

Recent developments have introduced a new way of acquiring phase information across the sound field with scanning techniques by using an additional acoustic sensor [15]. This allows applying transfer path analysis taking into account the phase relation between different sound sources. The results found so far proved that this new approach works remarkably well in laboratory conditions for mid-high frequencies. This paper presents the new methodology required for applying "Scan \& Paint TPA" to complex measurement scenarios, such as assessing the panel noise contribution of a car interior. Furthermore, advantages and disadvantages of the measurement technique are discussed considering its theoretical and practical limitations.

\section{THEORY}

In order to assess the underlying theory behind panel contribution analysis into a car interior, a general approach can be taken. Let us start defining a cavity $\mathbf{S}$ which surface excites the sound field when it is under operating conditions. Then, an infinitesimal small area $\mathbf{M}$ can be defined inside $\mathbf{S}$ for studying how different areas of the cavity 'contribute' to a point at M. Figure 1 shows a sketch of the scenario described above.

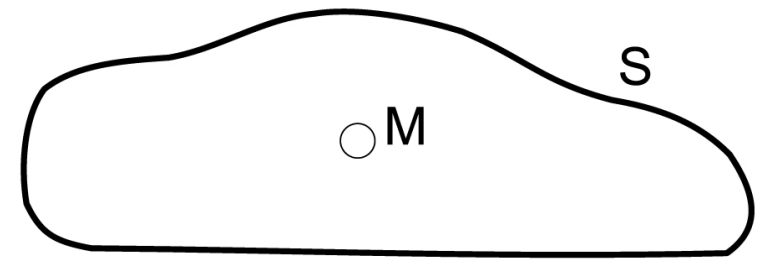

Figure 1. Schematic overview of the surfaces involved in the derivation

The theoretical derivations of an expression for calculating the pressure contribution at $\mathbf{M}$ follow Hald [7, 11$]$ and Kinsler [16]. First of all, it is necessary to define two different measurement conditions: when a monopole source at $\mathbf{M}$ is exciting the sound field (reciprocal transfer function measurements); and when the monopole is switched off and the cavity $\mathbf{S}$ is producing the noise (noise measurements).

Two sets of variables can be distinguished depending on the measurement conditions. $p^{T F}$ and $u^{T F}$ are defined as the pressure and particle velocity during the reciprocal transfer function measurements. On the other hand, $p$ and $u$ are the pressure and particle velocity during the noise measurements.
As have been pointed out by Hald, for deriving an expression which describes the fundamental relation of panel noise contribution analysis it is necessary to start using the definition of acoustic reciprocity [16],

$$
\int_{\mathbf{M}}\left(p^{T F} u-p u^{T F}\right) \mathrm{d} \mathbf{M}+\int_{\mathbf{S}}\left(p^{T F} u-p u^{T F}\right) \mathrm{d} \mathbf{S}=0
$$

The integral of particle velocity across the entire surface $\mathbf{M}$ will be zero due to there is no net energy going throughout $\mathbf{M}$ during the noise measurements. Furthermore, the pressure $p$ can be integrated over $\mathbf{M}$ during the noise measurements obtaining the reference pressure $p_{r}$. Besides, integrating the particle velocity over $\mathbf{M}$ during the transfer function measurement will lead to obtain the volume velocity of the monopole source $Q$. This leads to

$$
-p_{r} Q+\int_{\mathbf{S}}\left(p^{T F} u-p u^{T F}\right) \mathrm{d} \mathbf{S}=0
$$

The interior surface of the car can be assumed acoustically rigid for low frequencies [17]. This implies that the normal velocity of $\mathbf{S}$ is nearly zero during the transfer path measurement. Based on this assumption, Equation 2 simplifies to

$$
p_{r}=\int_{\mathbf{S}} \frac{p^{T F}}{Q} u \mathrm{~d} \mathbf{S}
$$

Equation 3 presents the base equation of most velocity-based panel noise contribution methods for mid-low frequency analysis. It relates the pressure at the reference position $p_{r}$ with the combination of particle velocity $u$ and acoustic transfer functions $p^{T F} / Q$ measured across $\mathbf{S}$.

So far, arbitrary signals have been considered on the derivation but for real scenarios it would be necessary to deal with random signals [18]. Moreover, Equation 3 cannot be used directly with scanning measurements because the source velocities are recorded one-by-one during a scanning such that the phase differences between source velocities at different points are unknown. To solve these problems, Equation 3 is rewritten firstly multiplying by the complex conjugate version of the pressure reference $p_{r}^{*}$ and then taking the expected values $E()$ of the different terms that could be treated as random variables, hence

$$
E\left(p_{r} p_{r}^{*}\right)=\frac{1}{A_{m}} \int_{\mathbf{S}} \frac{p^{T F}}{u_{m}} E\left(u p_{r}^{*}\right) \mathrm{d} \mathbf{S}
$$


where $u_{m}$ is the particle velocity at $\mathbf{M}$ during the transfer path measurements; and $A_{m}$ is the area of $\mathbf{M}$. Next, Equation 4 can be expressed by a combination of auto spectras and crossspectras, i.e.

$$
S_{p_{r} p_{r}}=\frac{1}{A_{m}} \int_{\mathbf{S}} \frac{S_{p^{T F} u_{m}}}{S_{u_{m} u_{m}}} S_{u p_{r}} \mathrm{~d} \mathbf{S}
$$

where $S_{\text {prpr }}$ is the autospectrum of the pressure reference; $S_{p} T F_{u_{m}}$ is the cross spectrum between the pressure at $\mathbf{S}$ and velocity at $\mathbf{M}$ both during the transfer function measurements; $S_{u_{m} u_{m}}$ is the autospectrum of $u_{m}$; and $S_{u p r}$ is the cross-spectrum between velocity at $\mathbf{S}$ and the reference pressure.

In practical cases, the surface $\mathbf{S}$ has to be discretized by dividing it into a limited number of panels N. Consequently, Equation 5 leads to

$$
S_{p_{r} p_{r}}=\frac{1}{A_{m}} \sum_{n=1}^{N} \frac{S_{p^{T F} u_{m}}}{S_{u_{m} u_{m}}} S_{\left(u p_{r}, n\right)} A_{n}
$$

where $A_{n}$ defines the area of each panel $n$.

\section{INSTRUMENTATION}

All measurements were carried out using a Microflown PU probe which contains a pressure microphone along with a particle velocity sensor. Furthermore, a GRAS random incidence microphone was used for measuring the reference pressure at the driver's ear. A Microflown low frequency monopole sound source was utilized to perform the reciprocal transfer function measurements. In addition, 12 cameras "Logitech Webcam Pro 9000" were required for recording all different sections of the car interior.

\section{METHODOLOGY}

The goal of the measurement procedure is to be able to localize and rank the dominant noise sources within a spectral region of interest. For this purpose, two main issues have to be addressed separately: noise sources and acoustic environment. The pressure at the reference position will be caused by the combination of how much noise the panels are inputting into the acoustic environment and how the environment itself affects the sound radiated. This statement can be inferred from the theoretical bases introduced in Section 2. Following this principle, the measurement procedure can be split into two parts: reciprocal transfer function measurements and particle velocity measurements.

\subsection{RECIPROCAL TRANSFER PATHS}

During the first stage, the noise sources under assessment must be switched off. Then the sound field is excited with a monopole source at a reference position. A low frequency monopole source was used along with a particle velocity sensor as a reference (see Figure 2) while pressure was measured scanning the car interior surfaces. Frequency limitations of reciprocal transfer function measurements are constrained by the effective working range of the monopole. Most panel contribution methods are not suitable for assessing low frequency problems. In order to demonstrate that "Scan \& Paint TPA" can be applied even in such a challenging frequency region, a monopole source with an effective frequency range from $30 \mathrm{~Hz}$ to $500 \mathrm{~Hz}$ was used.

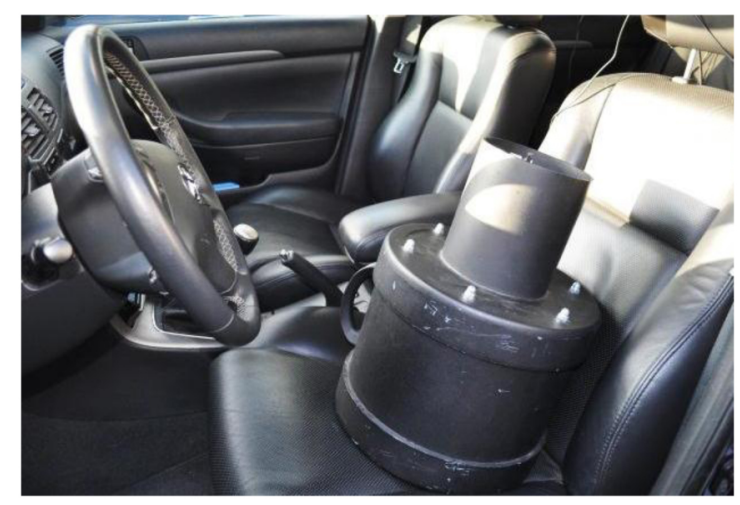

Figure 2. Low frequency monopole source

Scanning measurement techniques conventionally require time stationary conditions in order to evaluate different points of the sound field homogenously. Consequently, the monopole source was driven with random white noise band pass filtered between $20 \mathrm{~Hz}$ and $500 \mathrm{~Hz}$.

"Scan \& Paint" is a sound mapping technique based on mixing sound variations across a sound field with the relative position information of the probe extracted from a video. For simple scenarios were the excitation sources are within one visible plane, one camera angle is enough to follow the probe position during the whole measurement. However, a car interior is a complex case where one camera angle is not enough. Even if using a $360^{\circ}$ angle camera, some parts would be hidden. Moreover, the camera should be as perpendicular as possible to the measured plane in order to reduce any optical errors due to the projection on the 2D picture (video frames) [19]. From the previous it is evident that each vehicle interior requires different cameras angle distribution, depending on its internal dimensions and configuration.

In this paper a "Toyota Avensis" was studied with 12 camera angles distributed as follows: rear and front doors (4 cameras); dashboard, front window and front floor (1 camera); back floor ( 1 camera); ceiling (2 cameras); trunk sides and bottom (3 cameras); and back window (1 camera). Due to the fact that there is no global coordinate system established (the probe position is always relative to the background image), the cameras should be fixed during the 
testing process. Good fixing is essential for the successful combination of velocity and transfer functions measurements. Figure 3 shows a picture of the measurement setup with all the cameras distributed around the car interior.

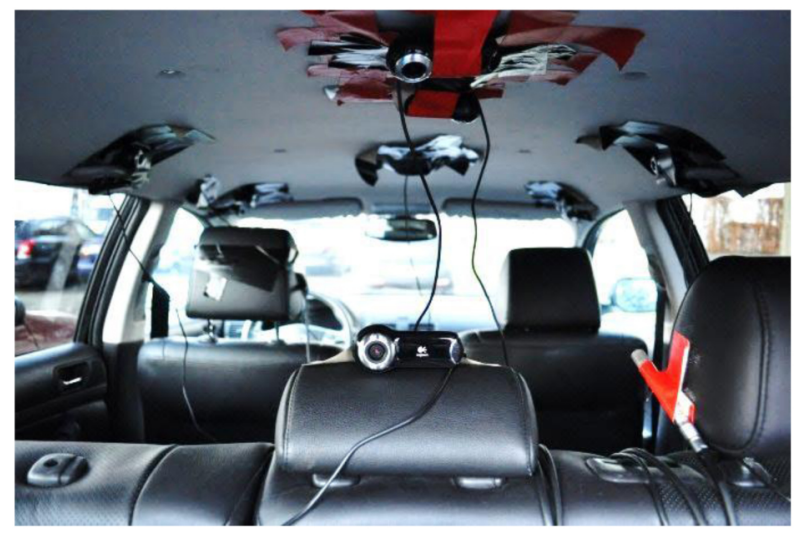

Figure 3. Measurement setup with 12 fixed cameras

Once all the cameras are fixed they will be used individually for recording the different car sections while acquiring transfer functions from the reference volume velocity source to the pressure nearby the surface. In order to evaluate different passenger spots it would be necessary to place the monopole in each position and scan again all the surfaces. In this paper only one listener position was assessed. The time needed for acquiring the transfer path data was 3 hours.

\subsection{PARTICLE VELOCITY}

Similar to the standard Scan \& Paint $[\underline{12}, \underline{13}, \underline{14}]$, the particle velocity in operational conditions is measured by scanning the surface with a PU probe. Again, due to the limitations of conventional scanning techniques, time stationary conditions are needed for performing the measurements. In the case studied, the measurements were carried out in a highway with a fixed speed of $80 \mathrm{~km} / \mathrm{h}$. Unwanted events (such as truck passing by) were avoided in the post-processing stage by evaluating the spectrogram of the fixed transducer.

Similarly to the reciprocal transfer function measurements, each individual section was recorded from its corresponding camera angle performing sweeps with a PU probe close the surface. The difference between conventional "Scan \& Paint" is that, relative phase information of the different sections is required. This issue is solved by using a reference microphone at the listener's position to have a fixed phase reference for all measurements.

In addition, other reference microphones would be required for studying the pressure contribution of multiple passenger positions. Nonetheless, it is important to take into account that the transfer function should be acquired with a monopole placed in each studied spot.

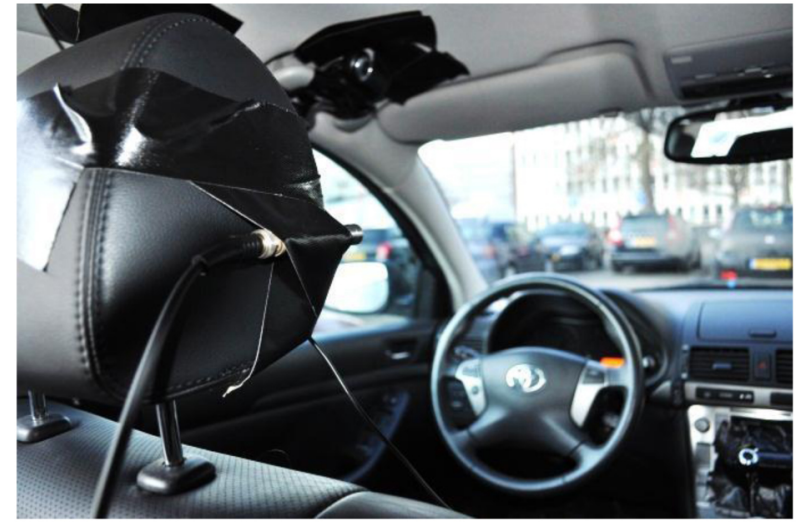

Figure 4. Reference pressure microphone at the driver's ear

\section{RESULTS}

Experimental results have been divided into four sections. First of all, graphs related to the validation of the measurement method are given. Secondly, some examples of velocity, transfer function and pressure contribution maps are shown. Two different car sections were chosen for studying their behavior throughout three different analyses. The frequency region assessed with the maps has been defined between $90 \mathrm{a} 100 \mathrm{~Hz}$, so as to evaluate what is happening with the different panels where the reference pressure microphone presents a powerful maximum in the low frequency region.

\subsection{METHOD VALIDATION}

Many different techniques based on transfer path analysis estimate the sound pressure level at a listener's position considering source strengths and transfer paths. Figure 5 presents a direct comparison between measured and estimated pressure, which is commonly the way of determining the validity and limitations of the measurement procedure. This figure certainly provides a clear evidence of the successful performance of the measurement methodology.

In order to understand the slight mismatching at higher frequencies Figure 6 presents the measurement dispersion between different sections. As can be seen, the reference pressure varies significantly at higher frequencies.

Figure 7 illustrates how the absolute error varies across the spectra along with the variance of the pressure dispersion shown in Figure 6. 


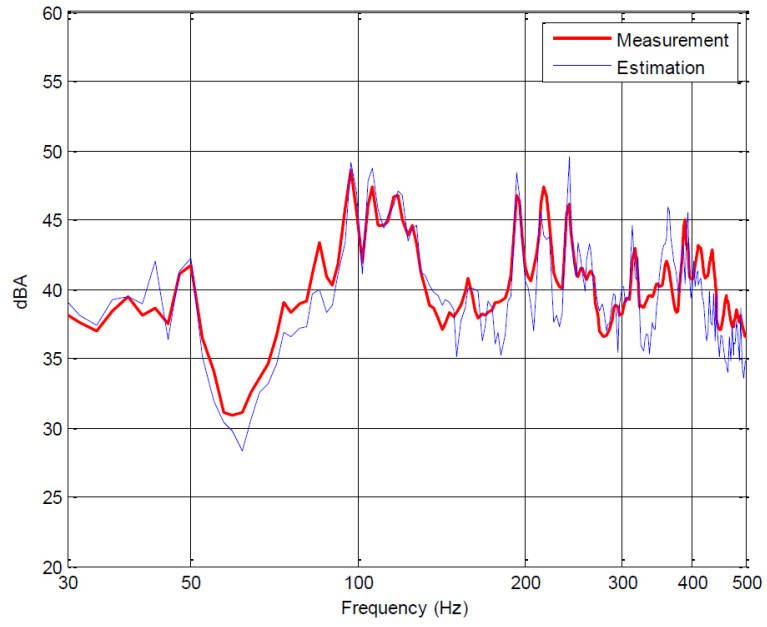

Figure 5. Measured and estimated reference pressure

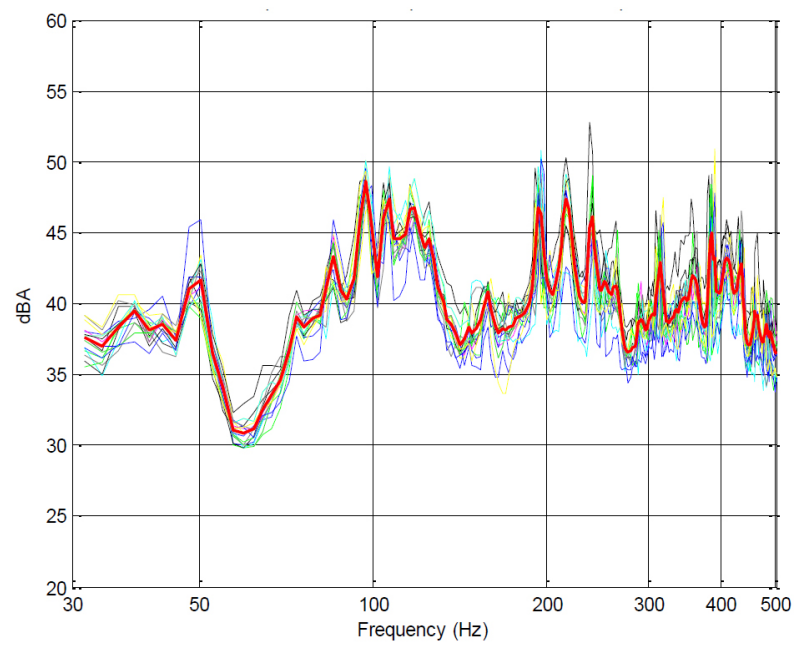

Figure 6. Pressure dispersion of the reference microphone during all different sections measured

As it is shown, the dispersion of the measurements is directly related with the estimation error found above $100 \mathrm{~Hz}$. Lack of stationary between different section measurements will lead to increase the error when all sections are added together for synthesizing the pressure at the reference position. In contrast, the error at lower frequencies does not depend on the variance between measurement sessions. The use of scanning techniques implies acquiring short time series which spectral estimation suffers from bias errors, especially at lower frequencies. Despite of this fact, results show that the error is reasonably good even at the lowest frequencies.

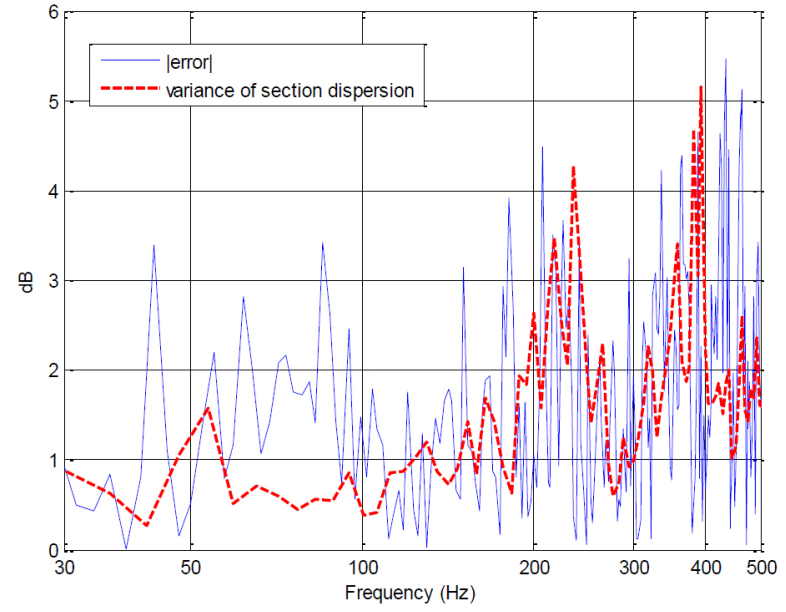

Figure 7. Absolute value of the error between measured and estimated pressure (blue) and variance of the reference pressure between different section measurements (red)

\subsection{VELOCITY MAPS}

Scanning particle velocity inside a car interior is a powerful way to detect smaller structural and airborne acoustic leakages [20]. Measuring the normal particle velocity close to a radiating surface gives a direct feedback on location of noise sources and characterization of vibrating patterns. However, particle velocity mapping should be assessed carefully because identifying potential problems in the near field does not necessary imply that they significantly contribute to the reference pressure. Figure 8 and Figure 9 presents the velocity maps of front right door and ceiling, respectively. As can be seen in Figure 8, there is a significant velocity maxima in the front part of the door.

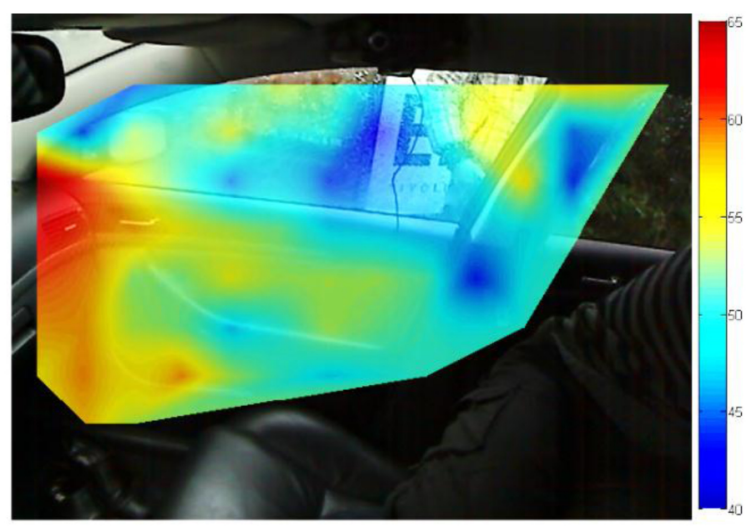

Figure 8. Narrow band (90-100 Hz) particle velocity mapping of the right front door (dB)

On the other hand, the narrow band map of the ceiling shows a clear symmetric pattern (Figure 9). The modal response of the structure becomes dominant at this critical resonance frequency band (see Figure 5). 


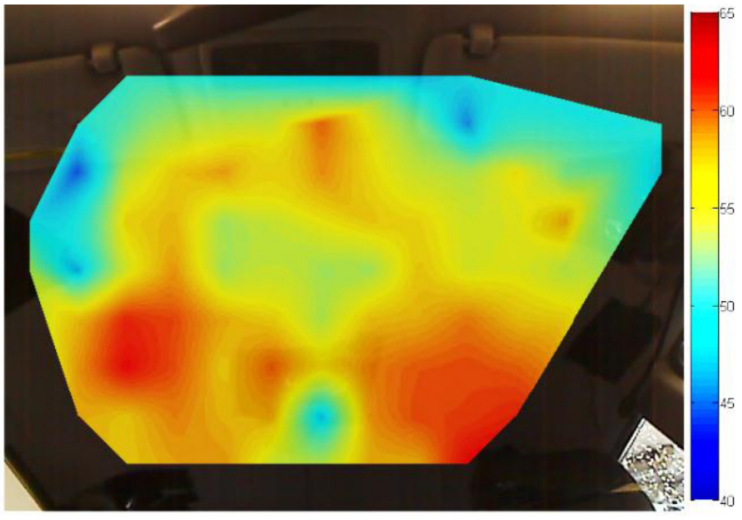

Figure 9. Narrow band $(90-100 \mathrm{~Hz})$ particle velocity mapping of the ceiling (dB)

\subsection{TRANSFER FUNCTION MAPS}

Acoustic transfer functions are useful to extract information of the measurement environment. It is important to know how the noise generated at the panels is modified when travels through the enclosure. Areas with high particle velocity do not necessarily contribute significantly to the pressure at the listener's position.

Figure 10 and Figure 11 shows the transfer function maps of the ceiling and front right door between 90 and $100 \mathrm{~Hz}$. As expected there is a transfer function maximum just on the top of the listener's position due to the proximity of the monopole source (Figure 10). Moreover, the level of surrounding areas decays smoothly as we get further away.

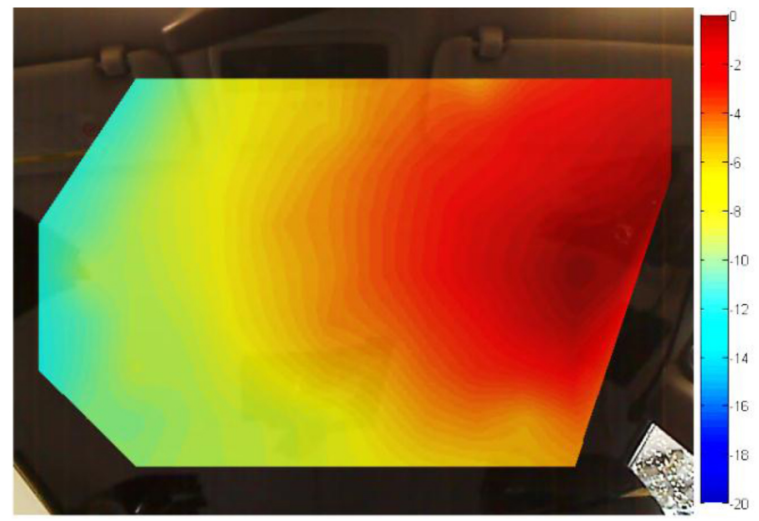

Figure 10. Narrow band (90-100 Hz) acoustic transfer function mapping of the ceiling(dBFS)

On the other hand, Figure 11 shows an interesting effect at the bottom corner of the door. This area seems to have an amplification effect due to its proximity with the open cavity between dashboard and floor. This example illustrates how certain areas can be magnified due to resonances of the car interior.

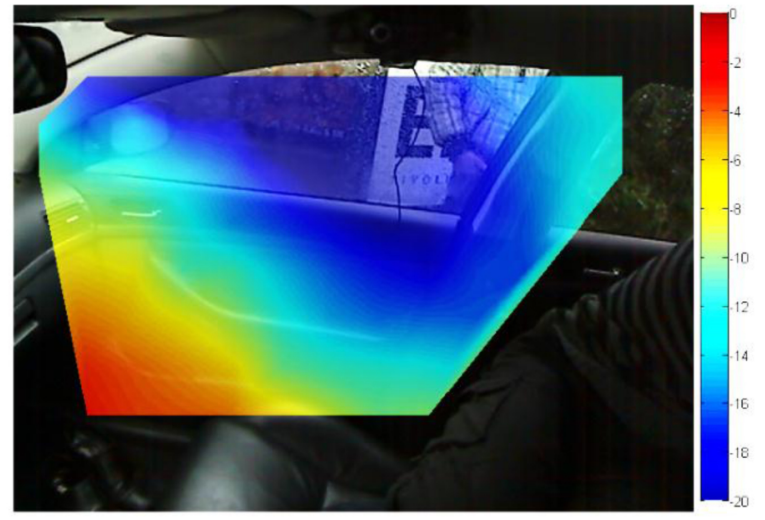

Figure 11. Narrow band $(90-100 \mathrm{~Hz})$ acoustic transfer function mapping of right front door (dBFS)

\subsection{PRESSURE CONTRIBUTION MAPS}

When the noise is aimed to be reduced at a certain spot it is essential to define the pressure contribution from different panels to rank the dominant sources. The ranking will give a direct understanding about which panels should be modified first.

Figure 12 and Figure 13 provides pressure contribution maps for ceiling and right front door. In the first case, Figure 12 does not present a symmetrical pattern as the one seen in Figure 9. Combining the surface excitation with the acoustic transfer functions the closer areas to the reference position becomes more relevant for the reference pressure. Even the effects of areas with lower excitation in the right hand side had been fairly magnified.

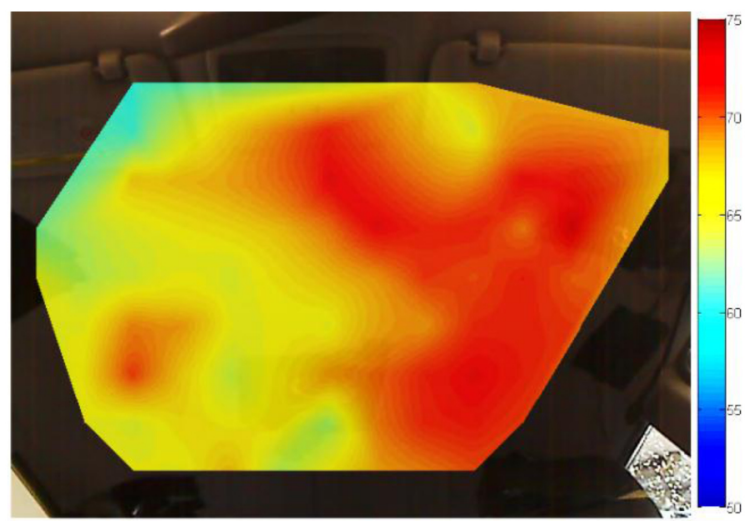

Figure 12. Narrow band $(90-100 \mathrm{~Hz})$ pressure contribution mapping of the ceiling (dBA)

Evaluating the right front door (Figure 13), can be seen that this section does not play such an important role in the pressure contribution. Even though it is clear that the area closer to the dashboard is the most problematic, the absolute levels are about $6 \mathrm{~dB}$ lower than the ceiling panels. Therefore, enhancing the right door will not decrease the 
pressure at the listener position. Ceiling is the dominant section that is masking other weak sources.

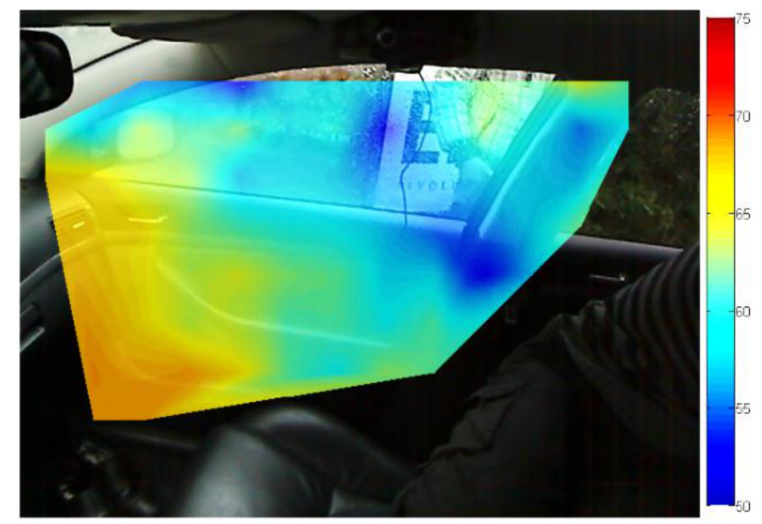

Figure 13. Narrow band (90-100 Hz) pressure contribution mapping of the right front door (dBA)

\section{DISCUSSION}

Advantages and limitations of the novel measurement technique "Scan \& Paint TPA" for assessing noise problem in car interior are described. Besides, future developments required in order to improve the performance of the method are stated.

\subsection{ADVANTAGES}

One of the main problems of conventional panel noise contribution techniques is the time required to perform the measurements and post process the data. However, manual sweeps of a single probe are a much faster procedure for directly obtaining the information required. Current "Scan \& Paint TPA" methodology requires only 1 day for carrying out reciprocal transfer function and noise measurements.

Cost of a measurement system is always a big issue. Most of the current multi-channel applications have far higher requirements against a one-probe solution.

Not only the measurement protocol but also the post processing stage is fairly intuitive. The use of multiple cameras make sure that almost all areas are captured and the measurements are filmed which proved to be helpful with trouble shooting. Color maps overlaid on pictures give a direct feedback that is easy to understand.

The amount of information obtained after the measurements allow to study in detail any single element of a car interior. Furthermore, the high spatial and frequency resolution of the results could only be compared with step-by-step measurements which are vastly more time consuming.
The main outcome of a measurement technique is to be able to ensure accuracy. The low error presented in the comparison between measured and synthesized pressure demonstrate the great potential of combining velocity-based scanning techniques with airborne transfer path analysis. Fixed point measurements suffer from discretization errors, choosing a fixed position to measure is always a risk of miss a source or leakage.

\subsection{LIMITATIONS AND FUTURE DEVELOPMENT}

One of the main problems of conventional scanning techniques is that time stationary conditions are required. Although some industrial applications are focused on transient or impulsive noise, most problems can be solved using stationary conditions.

Human errors such as touching the surface or producing noise while the probe is moving are inherent to the measurement technique. Nevertheless, they can be detected and avoided during the post processing stage.

It is important to point out that contribution maps presented in Section 5.4 were obtained by multiplying individual panel surface velocities by its corresponding transfer function according to Equation 6 (for $\mathrm{N}=1$ ). This method to produce the contribution maps does not consider any relation between different panels. Therefore, even though the color maps allow to visualize directly areas with high levels, correlated panels could be decreasing the effects shown in the map. Correlation relationships are taken into account when the global pressure synthesized is computed due to all panel contribution are integrated. Further research will to be focused on creating new visualizing method for producing contribution maps which also regard these effects.

Because the method does not measure the absolute probe position, there is only $2 \mathrm{D}$ information related to the background image which could lead to position errors for irregular surfaces. Furthermore, some panels (such as the driver's foot area) could not be measured because there was no direct vision during the noise measurements.

\section{CONCLUSIONS}

The new measurement technique "Scan \& Paint TPA" has been successfully validated for car interior assessment under stationary road conditions.

The good matching between pressure measured at the listener's position and the estimation provide clear evidence of the measurement success. It is important to highlight the good agreement at lower frequencies, which most conventional measurement methods are not able to assess. 
Surface velocity maps are useful for studying the volume velocity distribution across an enclosure surface. On the other hand, transfer function mapping allows seeing natural amplification or attenuation within an acoustics environment. Nevertheless, a pressure contribution mapping is required in order to find a clear method of ranking which sources are contributing most to a reference position.

\section{AKNOWLEDGEMENT}

The authors would like to thank HAN Automotive in Arnhem for kindly collaborating with this paper allowing to measure in their facilities.

\section{REFERENCES}

1. Klingenberg, H., "Automobil Messtechnik, Band A: Akustik," Springer, 1991

2. Pätzold, H. and Wedermann, G., "DIAMONDS Predictive Optimisation and Auralisation of LightWeight Absorption Sound Packages," Rieter Automotive Conference (2001/1)

3. Fahy, F. J., "Sound Intensity," E \& FN Spon, London, 1995.

4. Wolff, O., Guidati, S., Sottek, R., Steger, H., "Binaural auralization of vibrating surfaces," CFA/DAGA, 2004

5. Meyer, J., "Beamforming for circular microphone array mounted on spherically shaped objects,"”, J. Acoust. Soc. Am. 109 (1), 185-193, 2001.

6. Hald, J. and Tsuchiya, Masaki, "Panel Contribution Analysis using a Volume Velocity Source and a Double Layer Array with the SONAH Algorithm," In the proceedings of Internoise 2006.

7. Williams, E. G., "Fourier Acoustics. Sound Radiation and Nearfield Acoustical Holography," Academic Press, London, 1999

8. Verheij, J. W., "Inverse and Reciprocity Methods for Machinery Noise Source Characterization and Sound Path Quantification," International Journal of Acoustics and Vibration, 1997, 2(1), pages 11-20

9. Tijs, E., Wind, J., and Fernández Comesaña, D., "Fast High Resolution Panel Noise Contribution Method," SAE Technical Paper 2011-01-1594, 2011, doi: 10.4271/2011-01-1594.

10. Bertolini, C., Horak, J., Mantovani, M., Sinno, G. Lo, "Interior panel contribution based on pressure-velocity mapping and acoustic transfer functions combined with the simulation of the sound package," in Internoise 2011, Osaka, Japan, 2011.

11. Hald, J., "STSF - a unique technique for scan-based Near-field Acoustic Holography without restrictions coherence,” Brüel \& Kjær, Technical Review No., 1989.

12. Tijs, E., de Bree, H.-E., Steltenpool, S., "A novel acoustic pressure-velocity based method to access acoustic leakages of an acoustic enclosure in non anechoic conditions," in Euronoise 2009, Edinburgh, UK, 2009.

13. Tijs, E., de Bree, H.-E., Steltenpool, S., "Scan \& Paint: a novel sound visualization technique," in Internoise 2010, Lisbon, Portugal, 2010.

14. de Bree, H.-E., Tijs, E., Wind, J., Grosso, A., "Scan\&Paint, a new fast tool for sound source localization and quantification of machinery in reverberant conditions," in VDI Maschinenakustik, Lisbon, Portugal, 2010.

15. Fernández Comesaña, D. and Wind, J., "A Scanning Method for Source Visualization and Transfer Path Analysis Using a Single Probe," SAE Technical Paper 2011-01-1664, 2011, doi:10.4271/2011-01-1664.

16. Kinsler, L.E., Frey, A.R., Coppens, A.B. and Sanders, J.V., "Fundamentals of Acoustics," 4th edition.Wiley and Sons, 2000.

17. Grosso, A., Comesaña, D. Fernández, de Bree, H.-E. and Zajamsek, B., "Further development of the PNCA: New panel noise contribution reference-related (PNCAR)," in ISNVH 2012, Graz, Austria, 2012.

18. Percival, D.B. and Walden, A.T., "Spectral Analysis for Physical applications," Cambridge University Press, 1993.

19. Comesaña, D. Fernandez., "Mapping stationary sound fields," Master's thesis, Institute of Sound and Vibration Research, December 2010.

20. Grosso, A., De Bree, H., Steltenpool, S., and Tijs, E., "Scan and Paint for Acoustic Leakage Inside the Car," SAE Technical Paper 2011-01-1673, 2011, doi: 10.4271/2011-01-1673.

\section{CONTACT INFORMATION}

Daniel Fernandez Comesaña

Microflown Technologies

Tivolilaan 205

6824 BV Arnhem

the Netherlands

fernandez@microflown.com 
The Engineering Meetings Board has approved this paper for publication. It has successfully completed SAE's peer review process under the supervision of the session organizer. This process requires a minimum of three (3) reviews by industry experts.

All rights reserved. No part of this publication may be reproduced, stored in a retrieval system, or transmitted, in any form or by any means, electronic, mechanical, photocopying, recording, or otherwise, without the prior written permission of SAE. ISSN 0148-7191
Positions and opinions advanced in this paper are those of the author(s) and not necessarily those of SAE. The author is solely responsible for the content of the paper.

SAE Customer Service:

Tel: 877-606-7323 (inside USA and Canada)

Tel: 724-776-4970 (outside USA)

Fax: 724-776-0790

Email: CustomerService@sae.org

SAE Web Address: http://www.sae.org

Printed in USA 